\title{
COMPARATIVE STUDY BETWEEN OSTEOSYNTHESIS IN CONVENTIONAL AND BIOABSORBABLE IMPLANTS IN ANKLE FRACTURES
}

\author{
Guilherme Pelosini Gaiarsa ${ }^{1}$, Paulo Roberto dos Reis ${ }^{1}$, Rames Mattar Junior ${ }^{1}$, Jorge dos Santos SilvaA ${ }^{1}$, Túlo Diniz Fernandez ${ }^{1}$
}

\section{ABSTRACT}

Objective: To compare the functional results of ankle fractures treated with metallic and absorbable plates. Twenty patients were randomized into two groups (metallic and absorbable implant groups) and followed prospectively. In the immediate postoperative period, patients were immobilized with plaster casts for one week, which was replaced by a removable cast for another four weeks. Partial weight-bearing was allowed after three weeks, and full weight-bearing after six weeks. Functional recovery was similar in both groups. At six months, three patients in the metallic group complained of local pain, and had their implants removed. One patient in the absorbable group exhibited early dehiscence of the suture and underwent debridement and suturing with good evolution. The American Orthopaedic Foot and Ankle Society (AOFAS) score was similar between the two groups after six and nine months of follow-up. The absorbable implants showed clinical and functional results that were similar to those of metallic implants. Level of Evidence II, Prospective Comparative Study.

Keywords: Absorbable implants. Ankle injuries. Bone screws. Fractures, bone. Fracture fixation, internal.

Citation: Gaiarsa GP, Reis PR, Mattar Junior R, Silva JS, Fernandez TD. Comparative study between osteosynthesis in conventional and bioabsorbable implants in ankle fractures. Acta Ortop Bras. [online]. 2015;23(5):263-7. Available from URL: http://www.scielo.br/aob.

\section{INTRODUCTION}

Ankle fractures are very common, with a prevalence of 154 fractures per 100,000 inhabitants per year. ${ }^{1}$ It is estimated that between 30 and $40 \%$ of metal osteosynthesis performed are removed after fracture healing. The bioabsorbable osteosynthesis are an option to avoid the high rate of removal of synthetic material.

The aim of the study was to compare the outcomes of patients undergoing osteosynthesis of unstable simple line ankle fractures, through and above the tibiofibular syndesmosis, using bioabsorbable plate or conventional metallic implant, evaluating the surgical time, the loss of reduction, the need for removal of the synthesis material and the functional score after 6 and 9 months of surgery.

\section{CASES AND METHODS}

Patients diagnosed with uni- or bimalleolar ankle fractures classified as Weber B or C were selected. All patients were seen and treated at the Institute of Orthopedics and Traumatology, Hospital das Clínicas, São Paulo, SP, Brazil and Orthopedics Service of Hospital Municipal Dr. Moyses Deutsch, M'Boi Mirim,
São Paulo, SP, Brazil, from July 1, 2009 to August 1, 2010. All patients were duly informed about the necessity of surgery, the purpose of the study, its methods, both treatment options and randomization method. All patients signed a Free and Informed Consent form, as approved by the Ethics Committee of HC-FMUSP - CAPPesq under N 0108/09 on June 3, 2009.

We adopted the following inclusion criteria: patients aged between 18 and 60 years of both genders, with isolated simple line ankle fractures and indication for surgery (Weber types B or C), without associated dislocation (at the time of trauma), without clinical comorbidities that might delay the surgery or rehabilitation process and no previous history of allergic or inflammatory reaction to sutures. Between July 1, 2009 and August 1, 2010, there were seen in both services 84 patients with ankle fractures classified as Weber B and C. Only 20 met the inclusion criteria and were randomized by lot between the two groups. One patient in the metallic group was excluded for loss of follow-up, who returned after one year, but was kept excluded due to the inability to perform functional assessments at six and nine months.

All the authors declare that there is no potential conflict of interest referring to this article.

\footnotetext{
1. Universidade de São Paulo, Faculdade de Medicina, Department of Orthopedics and Traumatology, São Paulo, SP, Brazil.

Work developed at Universidade de São Paulo, Faculdade de Medicina, Department of Orthopedics and Traumatology, São Paulo, SP, and Hospital Municipal Dr. Moyses Deutsch, Orthopedics Service, M'Boi Mirim, São Paulo, SP, Brazil.

Correspondence: Rua Dr. Ovídio Pires de Campos, 333, Cerqueira Cesar, 05403-010 São Paulo, SP, Brazil. guilherme.gaiarsa@gmail.com
} 
All patients were operated by one of the research surgeons, familiar with both techniques and with metal and absorbable implants. All patients were followed by the senior researcher and by rehabilitation service personnel. All patients were assessed by radiographs and functional testing at six and nine months postoperative.

The treatment procedure used in both groups was the same: surgery performed within 10 days after the fracture, hospital discharge on the first or second day after surgery with suropodalic cast immobilization, return after one week to assess the surgical wound, placement of removable suropodalic brace and early stimulation of active mobility.

After two weeks we conducted X-ray control, removal of surgical stitches and partial load (with crutches) was started; after four weeks another $\mathrm{x}$-ray control was performed and load progression was allowed until reaching full load at six weeks postoperative. We performed serial radiographs until the twelfth week. The evaluation by the AOFAS score was performed at 6 months and again at 9 months after surgery by two orthopedists evaluators, one in each hospital, who did not participate in the surgical procedures. The evaluators were unaware to which group the patient belonged.

All radiographs were evaluated by the main investigator and the loss of reduction compared to the immediate postoperative radiographs was measured and consolidation assessed. The loss of reduction was considered as present (deviations larger than $2 \mathrm{~mm}$ ) or absent (deviations under $2 \mathrm{~mm}$ ). Likewise, the consolidation was assessed as present (presence of bone trabeculae) or absent (interruption on bone trabeculae).

\section{Surgical Technique}

All patients underwent spinal anesthesia. We used a cushion under the buttocks on the side to be operated and a pneumatic tourniquet calibrated at $300 \mathrm{mmHg}$. We performed the usual antisepsis preparation with chlorhexidine degerming solution, followed by alcoholic chlorhexidine solution, placement of sterile drapes, and exsanguination with sterile Esmarch band.

The surgery was initiated by the lateral malleolus, with posterolateral incision, anatomical reduction with the aid of reduction clamps, and fracture fixation.

In the metal group we used compression interfragmentary screws with anti-slip posterolateral plate, or lateral neutralization. ${ }^{2}$

After synthesis of the fibula, we performed the synthesis of the medial malleolus (where there was this associated fracture) and assessed the integrity of the syndesmosis by direct tension maneuvering with Backhaus clamps. In cases where instability was verified, we used $3.5 \mathrm{~mm}$ tricortical screw fixing the fibula to the tibia just above the syndesmosis.

In the absorbable group synthetic material did not allow the use of interfragmentary compression screw. We use a positioning screw, followed by anti-slip posterolateral plate. The plate is initially set in the proximal fragment. After fracture reduction the plate is fixed using three distal and three proximal screws. The absorbable material is radiolucent, only drilling in bone was visible in radiographic image.

The absorbable plate is molded upon warming in saline bath $\left(70^{\circ} \mathrm{C}\right.$ for one minute). As required, we made new holes in the absorbable plate for syndesmosis fixation (five of six patients had unstable syndesmosis).
The surgical technique with absorbable material included a greater periosteum removal around the fracture to allow better shaping of the absorbable plate on the bone, upon warming it up. For insertion of absorbable screws we performed a previous fracture reduction, stabilization with reduction clamps, drilling and full tapping of holes.

We use oval shape, fusiform body absorbable implant INION $\AA$ (Finland) without sharp edges, with two rows of holes, with six or eight holes and a specific laterality. The plates used have a more prominent clipping in the back side, covering the lateral malleolus, without overlapping the groove of the fibular tendons. The screws used were 2.8 or $3.1 \mathrm{~mm}$ (outside diameter) used according to necessity. (Figure 1)

Placement of the $4.5 \mathrm{~mm}$ cannulated screw to secure the syndesmosis in instable cases ${ }^{3}$ was carried out off the plate (in case where the plate was out of the path) or through the plate (by a hole made through the plate, in 5 of 6 cases). Syndesmosis was fixed using a reduction clamp during the introduction of this screw.

In both techniques, we performed immediate postoperative radiographs prior to closure of the subcutaneous tissue and skin. In all patient we used after cast immobilization for a week after dressing, until substitution by the immobilizer (suropodalic brace).

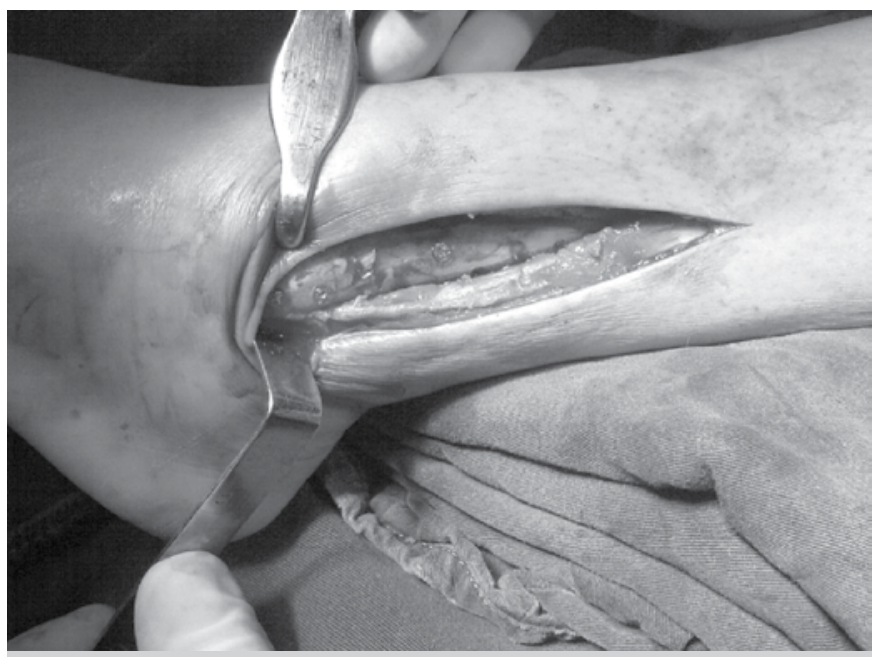

Figure 1. Absorbable plate in place.

\section{Statistical analysis}

Patients were included and distributed independent and randomly into groups, ensuring the impartiality of the results. Care was taken to reduce interference ("noise" or "bias") caused by uncontrollable variables by adopting specific inclusion criteria for the standardization of experimental groups (filters).

For description and analysis of patients gender (categorical variable) according to the type of implant (group or sample), an absolute $(\mathrm{N})$ and relative (\%) frequency distribution was held. Comparisons of class frequencies between groups (samples) for inference on the difference of occurrence frequency were made by Fisher's exact test.

For sample description and analysis of quantitative data (specific quantities or continuous variables), the patient's age (years old) at the time of surgery, the score of the functional classification (AOFAS) and intraoperative time (min), descriptive statistics was 
used: mean (M) and median (MN). In case of non-parametric samples we used standard deviation (SD), standard error of the mean (SEM), maximum (MAX) and minimum values (MIN) and number of cases $(\mathrm{N})$.

Normality of distributions was tested by Pearson's coefficient of variation (PCV) and the Kolmogorov-Smirnov test for continuous variables. Parametric distribution was assumed in cases where the Pearson coefficient of variation (PCV) was less than $30 \%$ or the Kolmogorov-Smirnov (KS) test showed non-significant result. Parametric tests were adopted only for comparisons in which the two distributions have been considered normal (Gaussian curve).

In comparisons between samples for inference of difference between mean values of variables, we used the Mann-Whitney Utest for independent samples and non-parametrically distributed and Student $t$-test for unrelated samples and parametric distribution. The adopted confidence level was $5 \%(\alpha=0.05)$.

It was previously assumed that the samples (groups) would be equivalent. Two dimensional or bilateral tests were used: $\left(\mathrm{H}_{0}=\mu_{1}-\mu_{2}=0\right)$

We used the statistical software GraphPad Prism ${ }^{\circledR}$ version 2.01 (1996) from GraphPad Software Incorporated ${ }^{\circledR}$ and tools supplement of data analysis (statistics) from Microsoft Excel 2007 ${ }^{\circledR}$ from Microsoft Corporation ${ }^{\circledR}$ (USA).

Of the 20 selected patients, twelve were female and eight male, aged 21-60 years who were randomized at the time of communication of surgery into metal group and absorbable implants group. Of the nine patients who underwent osteosynthesis with metal implant five (55.5\%) were male and four patients (44.4\%) were female. Of the 10 patients undergoing fixation with absorbable implant two (20\%) were male and eight (80\%) were female. The evaluation of the sample showed no statistically significant difference between groups.

The mean age of patients undergoing fixation with metallic implant was 38.9 years and those who underwent fixation with absorbable implant were on average 39.5 years old. The comparison between groups was not statistically significant, showing a homogeneous sample.

During surgery, four patients had minor problems with absorbable implants. In two of them it was necessary to use anti-rotational steel wires to fix fractures of the medial malleolus, since absorbable screws that allow compression have a $4.5 \mathrm{~mm}$ diameter (too large for use in medial malleolus fractures). In three patients, there was screw breakage during insertion, requiring new drill with a larger diameter drill, and switching to larger screw (2.8 $\mathrm{mm}$ to $3.1 \mathrm{~mm}$ diameter).

During evolution, there was a case of wound dehiscence in the first week, in a patient from the absorbable group. This patient evolved satisfactorily with surgical cleaning, debridement and new suture with no need for removal of synthesis and without further complications.

Three patients of the metallic implant group underwent removal of synthesis material due to local pain. One of them had an eversion ankle restriction. Another presented local pain to palpation and a third one evolved, after load bearing was allowed, with release of the syndesmosis screw. The latter had local pain and a small wound caused by screw prominence.

A patient of the absorbable group, although evolving without loss reduction showed bone callus hypertrophic to X-ray at Acta Ortop Bras. 2015;23(5):263-7
12 weeks (hypertrophic callus consolidation), indicating that synthesis was not rigid.

All patients were considered consolidated in the evaluation at 12 weeks and none was consolidated at eight weeks. Therefore, all consolidation occurred between the $8^{\text {th }}$ and $12^{\text {th }}$ weeks, and there was no reduction loss.

Among patients submitted to synthesis with metallic implants, the average AOFAS score at six months was 83.7, while at the ninth months it was $89.9(p=0.08)$, the group submitted to synthesis with absorbable implants the average score at six months was 87.1, while at nine months it was $88.1 \quad(p=0.18)$, results considered not statistically different $(p=0.57)$. (Table 1 ) Surgical time, measured by the anesthesia form, was apparently higher in the absorbable group than in the metal group, with an average of $75 \mathrm{~min}$ in the metallic group versus $97.5 \mathrm{~min}$ in the absorbable group, a result considered not statistically significant $(p=0.16)$.

Table 1. Descriptive statistics of functional assessment score (AOFAS) according to implant material and time of implant (months). Interclass comparison by paired t-test after 9 months from implant, by the Student t-teste $(\alpha=0.05)$.

\begin{tabular}{|c|c|c|c|c|c|}
\hline \multirow{3}{*}{ AOFAS Score } & \multicolumn{5}{|c|}{ Implant material } \\
\hline & \multicolumn{2}{|c|}{ Metal } & \multicolumn{3}{|c|}{ Absorbable } \\
\hline & 6 months & 9 months & 6 months & \multicolumn{2}{|c|}{9 months } \\
\hline Mean & 83.7 & 89.9 & 87.1 & \multicolumn{2}{|c|}{88.1} \\
\hline Standard Deviation & 9.5 & 5.4 & 6.6 & \multicolumn{2}{|c|}{7.8} \\
\hline EPM & 3.2 & 1.8 & 2.1 & \multicolumn{2}{|c|}{2.4} \\
\hline MAX & 96 & 100 & 96 & \multicolumn{2}{|c|}{100} \\
\hline MIN & 63 & 83 & 74 & \multicolumn{2}{|c|}{74} \\
\hline $\mathrm{N}$ & 9 & 9 & 10 & \multicolumn{2}{|c|}{10} \\
\hline PCV (\%) & 11.39 & 5.98 & 7.61 & \multicolumn{2}{|c|}{8.82} \\
\hline K-S (p) & $>0.10$ & $>0.10$ & $>0.10$ & & 10 \\
\hline & & & & & \\
\hline Paired t-Test & & & & & \\
\hline & $t=1.96$ & $p=0.08$ & $t=1.46$ & & .18 \\
\hline & & & & & \\
\hline Student t-Test & & & & & \\
\hline & & & & & \\
\hline
\end{tabular}

\section{DISCUSSION}

Our motivation for this study was the availability of the implants, relatively new in our midst, and the frequent need for implants removal in ankle fractures. These fractures are very common, with a prevalence of 154 fractures per 100,000 inhabitants per year. ${ }^{2}$ It is estimated that $30-40 \%$ of the metal plates used for ankle osteosynthesis ought to be removed. ${ }^{4}$

In order to compare ankle osteosynthesis with metal plates and absorbable material, we selected patients with fractures classified as Weber types B and C. These fractures have similar clinical features and treatment indication. ${ }^{2}$ We used the Weber classification for its greater reproducibility. Weber B and C closed fractures have similar mechanisms of trauma and the prognosis only differ according to initial trauma, ${ }^{5}$ which factors are associated to poor prognosis: exposure of the fracture focus, presence of associated dislocation, impairment of all malleoli (trimalleolar fractures) and age 60 years or older. ${ }^{6}$ In our series all fractures were closed in patients below 60 years old, without associated dislocations. 
We evaluated and included patients from two hospitals that deal with highly complex traumas and multiple trauma patients. Because of the characteristics of these hospitals, we evaluated a large number of patients, but were able to include only 20 , as many had associated injuries, exposure of the fracture site and other comorbidities. The one year period for the selection and treatment of patients also influenced the sample size.

Simple randomization led to quite homogeneous groups in terms of age distribution, but with unequal distribution according to gender. The proportion of eight women and two men in the group submitted to osteosynthesis with absorbable material, although large, was not statistically significant. Anyway, considering the patients inclusion criteria, we do not believe that differences in gender frequency may have influenced our results. The fixation with metallic material followed the techniques described by Weber and Krause ${ }^{2}$ at the $\mathrm{AO}$ guidebook, with fracture reduction, stabilization with reduction clamps, fixation with interfragmentary screw and neutralization plate or anti-slip posterior plate with plate compression, since the results of both techniques are similar. ${ }^{4}$

Among patients undergoing treatment with metallic material, one was excluded for not having attended follow-up. Of nine patients followed three underwent removal of the synthesis material at around six months postoperatively. In two of them indication to remove synthetic material was based on complain of severe pain and hence, a poor functional outcome. One patient had inflammation in the fibular tendons in the topography of the posterolateral plate. Inflammation of fibular tendons is a common problem of posterolateral plates. This patient evolved well after the removal of the plate with one of the best functional scores at nine months (three months after plate removal).

Of the two patients who had undergone removal of metallic synthetic material due to pain, one patient showed adherence in the site of the lateral plate. Adherence and local pain, when the plate is used laterally, is also common. ${ }^{4}$ This patient showed improvement of pain upon the removal of the plate. In the other patient, the screw that fixed the syndesmosis came loose and migrated, instead of breaking, causing pain, local irritation and bloody area. After removal of the screw, the patient evolved with no pain.

In our series, the rate of synthesis metallic material removed was $30 \%$ in nine month follow-up, a rate consistent with the literature. The indication of removal of implants in all of them was pain, which improved within two weeks after removal of syndesmoses plates and screw. ${ }^{7,8}$

There are few scientific descriptions of the use of absorbable synthetic material in ankle fractures. The first report was carried out over 25 years ago using PGA (poliglicolic Acid) and PLA (polilactic Acid) flat pins.

The mechanical strength of the absorbable implants, although lower than that of metallic implants, was sufficient to stabilize these fractures. This resistance was also evaluated in metacarpal fractures and fixation of tibial-fibular syndesmosis. ${ }^{9}$

We used in this study a new generation of absorbable synthetic materials $\left(\mathrm{INION}^{\circledR}\right)$, polymer compounds which do not contain polyglycolic acid, accounted for most of the previously reported complications in the literature. ${ }^{10,11}$ The implants used are levorotatory and dextrorotatory compounds of the polylactic acid (PLLA/PDLA) and trimethylene carbonate (TMC), which are manufactured specifically for the ankle, being absorbed by hydrolysis within 18 months.
The surgery time was measured from the time of incision until dressing closing, and based on the anesthesia chart of the patients' records. Although not statistically significant, we observed a trend of surgical time to be higher in the absorbable implants group. Surgical time, when using absorbable material, is theoretically higher because the surgical technique should be more precise, requiring better removal of periosteum, the plate needs to be hot molded, it is necessary to measure and open the individual packaging of each screw and some screws have to be cut to obtain the desired length. These necessary procedures when using absorbable implants expend surgical time, which is not required in metallic synthesis.

The use of absorbable implants should be preceded by training with models, or cadaver bones, due to peculiarities of its use. Firstly, the reduction of the fracture with calipers must be perfect, since there is no compression by the screw. Then, drilling must be complete in both cortical, without any loss of reduction to allow for "tapping" of both cortical bones, for the introduction of the screw takes place without resistance.

Prior to the introduction of a screw, it should be moistened with physiological saline, and the orifice must also be washed with saline, to prevent the bolt from locking in the way and breaking. A distinctive sound and increased screw opacity alerts to imminent screw breakage, and the need for its immediate removal and improved preparation of the hole, in case the screw has not yet been fully inserted.

This difficulty occurs because of fragility in torsion, which is a characteristic of absorbable polymers and one of the difficulties in its use. ${ }^{4}$

Despite care provided, screws broke in three patients from the absorbable group during their placement, which has led to the need for new drilling with a larger drill, "tapping" and placement of a larger diameter screw. However, screw breaking is considered a minor complication which is easily resolved with the available instruments. However, this implant break and its replacement imply in increased costs and increased surgical time. These technicalities and complications are not described by Kukk and Nurmi ${ }^{12}$ using absorbable plates and screws, neither by authors who use only absorbable screws. ${ }^{8,13}$

Among the complications of the group submitted to synthesis with absorbable implant, we observed an early suture dehiscence in patients with severe swelling in the ankle during the surgery. This patient developed satisfactorily after surgical cleaning and new skin suture after three weeks. We did not relate dehiscence to the implant material.

A patient of the absorbable group, despite the good clinical and radiological outcomes, evolved with hypertrophic callus formation observed in the $12^{\text {th }}$ week radiography. This finding demonstrates a relative stability of the synthesis, where absolute stability was expected. While not leading to clinical repercussions, it was considered a minor complication.

The fact that patients of the absorbable group did not present fibular tendon irritation can be attributed to the design of these plates, spindle-shaped with rounded edges and their better shaping to the bone, a fact that prevents them from invading the tendon groove.

The evaluation through AOFAS questionnaire, in our study, was performed by two examiners who did not know to which group the patient belonged, being an examiner from each hospital 
where cases were operated and followed. Functional evaluation was performed six and nine months after surgery in all patients. In three patients of the metal group we observed worse results in the sixth month. These patients underwent removal of the synthesis material due to complaints of pain, and had their scores improved on the ninth month. This result was not statistically significant.

We decided to perform clinical evaluation at six and nine months, since we believe that at six months all fractures were consolidated, and we would have the opportunity to assess losses in reduction and their clinical correlation. At nine months we expected that patients were returning to their professional activities and we could better assess their recovery.

Our study shows great similarity in the results of both groups. On the radiographic point of view no patient had loss of reduction compared to the immediate postoperative image. On clinical outcomes at six and nine months, according to the functional score, the results were similar.

The rehabilitation process and load release for patients of the absorbable group was a major criticism of this synthesis, since we thought it would be necessary to delay the rehabilitation process due to the fragility of the material. We set out to accomplish the same protocol rehabilitation and load progression used by foot and ankle group of our institution, in both groups, in case this weakness occurred, we could observe it earlier. Kukk and Nurmi's ${ }^{12}$ report the need for delayed load progression in groups undergoing fixation with absorbable materials, while other authors keep all patients, regardless of the synthesis material, immobilized and unloaded for 6 weeks. We used removable brace from the second week on, with progressive load from the third week. We did not observe reduction loss in any patient.

The only study found that compares the use of absorbable plates and screws with metal plates and screws, is Kukk and Nurmi's, ${ }^{12}$ who retrospectively assessed patients treated with both types of implants, and considered similar results. Thus, prospective and randomized studies of these implants were not found to have a basis for comparison.

Kukk and Nurmi ${ }^{12}$ compared ankle osteosynthesis and syndesmosis with metal and absorbable material. None of the articles make clear whether the rehabilitation was similar in both groups and what the functional evaluation criteria were. We conducted a study on rehabilitation and similar load progression in both groups, with functional assessment at two different times, and found very similar results in both groups, more consistently than demonstrated in these studies.

Experience with absorbable materials, combined with other articles $^{14,15}$ allowed to devise a hybrid synthesis, where interfragmentary compression screws, which are not in contact with the subcutaneous tissue neither along to tendons, could be metallic, associated with neutralizing absorbable plates located in the places where the removal of implants is often necessary. This study has some limitations. The small sample size leads to great limitations in the statistical analysis. The use of a new implant, with its own characteristics, requires a learning curve that was not included in this study. The patient follow-up period may be insufficient for adverse reactions to absorbable materials to occur, despite being a prospective study and some cases had longer follow-up periods without complications.

The fact of being a comparative study allows difficulties with a new material to be more noticeable than in a case series.

The assessment by plain radiographs is limited both in the evaluation of consolidation, as in the reactions during absorption of the implants, however, the use of CT scan evaluation would be an unjustifiable exposure of patients to ionizing radiation, and serial MRI was out the technical and financial reach of this study.

\section{CONCLUSIONS}

The comparison of clinical outcomes by functional AOFAS score showed similar results in patients undergoing osteosynthesis with metal and absorbable implant material at six and nine months postoperative assessment.

The radiographic outcome, measured by loss of reduction was similar in both groups.

The need for further surgery to remove synthesis material was higher in the metal group.

Comparing the results obtained in both groups, we observed that the surgical time in the ankle osteosynthesis with absorbable material appears to be greater than when using metallic material, but not statistically significant in this study.

\section{REFERENCES}

1. Pakarinen HJ, Flinkkiala TE, Ohtonen PP, Ristiniemi JY, Stability criteria for nonoperative ankle fracture management. Foot and Ankle Int. 2011;32(2):141-7.

2. Weber M, Krause F. Peroneal tendon lesions caused by antiglide plates used for fixation of lateral malleolar fractures: The effect of plate and screw position. Foot Ankle Int. 2005;26(4):281-5

3. Cox S, Mukherjee DP, Ogden AL, Mayuex RH, Sadasivan KK, Albright JA, et al. Distal tibiofibular syndesmosis fixation: a cadaveric, simulated fracture stabilization study comparing bioabsorbable and metallic single screw fixation. J Foot Ankle Surg. 2005;44(2):144-51.

4. Muller ME, Allgower M, Schneider R, Willenegger $\mathrm{H}$. Malleolar fractures -manual of internal fixation - techniques recommended by the AO-ASIF. Berlin Heidelberg: Springer-Verlag; 1969.

5. Lauge-Hansen N. Fractures of the ankle. II. Combined experimental-surgical and experimental-roentgenologic investigations Arch Surg. 1950;60(5):957-85

6. Cox S, Mukherjee DP, Ogden AL, Mayuex RH, Sadasivan KK, Albright JA, et al. Distal tibiofibular syndesmosis fixation: a cadaveric, simulated fracture stabilization study comparing bioabsorbable and metallic single screw fixation. J Foot Ankle Surg. 2005;44(2):144-51.

7. Broos PL, Bisschop AP. Operative treatment of ankle fractures in adults: correlation between types of fracture and final results. Injury. 1991;22(5):403-6.

8. Joukainen A, Partio EK, Waris $\mathrm{P}$, Joukainen J, Kroger $\mathrm{H}$,Tormala $\mathrm{P}$, et al.

Acta Ortop Bras. 2015;23(5):263-7
Bioabsorbable screw fixation for the treatment of ankle fractures. J Orthop Sci. 2007;12(1):28-34

9. Kaukonen JP, Lamberg T, Korkala O, Pajarinen J. Fixation of syndesmotic ruptures in 38 patients with a malleolar fracture: a randomized study comparing a metallic and a bioabsorbable screw. J Orthop Trauma. 2005;19(6):392-5.

10. Bergsma JE, de Bruijn WC, Rozema FR, Bos RR, Boering G. Late degradation tissue response to poly(L-lactide) bone plates and screws. Biomaterials. 1995; 16(1):25-31.

11. Brown OL, Dirschl DR, Obremskey WT. Incidence of hardware-related pain and its effect on functional outcomes after open reduction and internal fixation of ankle fractures. J Orthop Trauma. 2001;15(4):271-4.

12. Kukk A, Nurmi JT. A retrospective follow-up of ankle fracture patients treated with a biodegradable plate and screws. Foot Ankle Surg. 2009;15(4):192-7.

13. Ahmad J, Raikin SM, Pour AE, Haytmanek C. Bioabsorbable screw fixation of the syndesmosis in unstable ankle injuries. Foot Ankle Int. 2009;30 (2):99-105

14. Lamontagne J, Blachut PA, Broekhuyse HM, O'Brien PJ, Meek RN. Surgical treatment of a displaced lateral malleolus fracture: the antiglide technique versus lateral plate fixation. J Orthop Trauma. 2002;16(7):498-502.

15. Winkler B, Weber BG, Simpson LA. The dorsal antiglide plate in the treatment of Danis-Weber type-B fractures of the distal fibula. Clin Orthop Relat Res. 1990;(259):204-9. 